\title{
Parity nonconservation in electron recombination of multiply charged ions
}

\author{
G. F. Gribakin' and F. J. Currell \\ Queen's University, Belfast BT7 1 NN, Northern Ireland, United Kingdom \\ M. G. Kozlov and A. I. Mikhailov \\ Petersburg Nuclear Physics Institute, Gatchina 188300, Russia
}

(Dated: March 23, 2022)

\begin{abstract}
We discuss a parity nonconserving asymmetry in the cross section of KLL dielectronic recombination of polarized electrons on the hydrogen-like ions with $Z \lesssim 60$. This effect is strongly enhanced because of the near-degeneracy of doubly-excited $2 l 2 l^{\prime}$ states of opposite parity in He-like ions. For ions with $Z \sim 30$ the asymmetry is of the order of $10^{-9}$. For $Z \approx 48$ a level crossing takes place, leading to the PNC asymmetry of $\pm 5 \times 10^{-9}$, which is $10^{8}$ times greater than the basic strength of the weak interaction in atoms.

PACS numbers: $32.80 . Y s, 34.80 . \mathrm{Lx}, 11.30 . \mathrm{Er}$
\end{abstract}

\section{INTRODUCTION}

Parity nonconservation (PNC) is caused by the weak interaction. According to the standard model this interaction is described in terms of charged and neutral currents. The charged currents play a dominant role in nuclei, e.g. in $\beta$-decay. The neutral currents lead to the PNC electron-nuclear interaction and can be observed in atomic experiments [1. In this paper we propose that enhanced PNC effects can be seen in electron recombination of multiply charged ions (MCI).

The first suggestions and estimates of PNC effects in MCI were made in 1974 by Gorshkov and Labzovskii [2]. A successful observation of PNC effects in optical experiments with heavy neutral atoms (see the recent review [3] for references) has renewed the interest in PNC effects in MCI 4, 5, 6, 7, 8, 9, 10. The obvious advantage of MCI is the $Z^{5}$ scaling of the PNC matrix elements with the nuclear charge $Z$, as opposed to $Z^{3}$ scaling in neutral atoms [1].

However, this advantage is usually compensated by larger energy differences between the levels of opposite parity. Indeed, PNC effects in atoms and ions appear because of the mixing of the levels of opposite parity. This mixing leads, for example, to an admixture of a negativeparity state $\psi_{-}$to a positive-parity state $\psi_{+}$due to the parity nonconserving weak interaction $H^{\mathrm{PNC}}, \psi_{+}+i \eta \psi_{-}$, as determined by the first-order perturbation expression

$$
i \eta=\frac{\left\langle-\left|H^{\mathrm{PNC}}\right|+\right\rangle}{E_{+}-E_{-}+\frac{i}{2} \Gamma_{-}} .
$$

The mixing coefficient $\eta$ is real when the level width $\Gamma_{-}$ is negligible compared to the level spacing $E_{+}-E_{-}$. In neutral atoms the valence energies are roughly independent of $Z$, and $\eta$ scales as $Z^{3}$. In MCI the level energies

\footnotetext{
*E-mail address: g.gribakin@am.qub.ac.uk

${ }^{\dagger}$ E-mail address: mgk@MF1309.spb.edu
}

$E_{ \pm}$are proportional to $Z^{2}$ and a typical PNC mixing $\eta$ again scales as $Z^{3}$.

In some special cases the levels of opposite parity in MCI can be anomalously close. For example, levels of the configurations $1 s 2 s$ and $1 s 2 p$ in He-like ions cross several times as $Z$ varies 2 . Their proximity leads to a strong enhancement of the PNC effects. At the crossing point $\left(E_{-}=E_{+}\right)$the maximal size of the mixing parameter is limited by the level widths and can be estimated as:

$$
\eta \sim\left\langle-\left|H^{\mathrm{PNC}}\right|+\right\rangle / \Gamma_{ \pm} .
$$

According to Ref. 2, a crossing of the $1 s 2 p{ }^{3} P_{1}$ and $1 s 2 s{ }^{1} S_{0}$ levels takes place at $Z \approx 32$. Because of the difference in the total electronic angular momentum, these levels can only be mixed by the nuclear-spin-dependent (NSD) part of the PNC interaction 9. Two oppositeparity levels with the total angular momentum $J=0$, $1 s 2 s{ }^{1} S_{0}$ and $1 s 2 p^{3} P_{0}$, cross twice at larger $Z$, around 65 and 90 [11. For such ions one can expect enhanced nuclear-spin-independent (NSI) PNC effects. In both cases the detection schemes involve radiative transitions.

In this paper we propose to study PNC mixing in Helike ions by looking at the parity-violating asymmetry in KLL dielectronic recombination (DR) of electrons with $\mathrm{H}$-like ions. Here the PNC interaction manifests itself as a difference between the recombination cross sections for electrons with positive and negative helicities. The observation of such difference means a correlation between the spin and momentum of the incident electron of the form $\boldsymbol{\sigma} \cdot \boldsymbol{p}$, which does violate parity, since $\boldsymbol{p}$ is a vector and $\boldsymbol{\sigma}$ is a pseudovector $(\boldsymbol{p} \rightarrow-\boldsymbol{p}$, while $\boldsymbol{\sigma} \rightarrow \boldsymbol{\sigma}$ under spatial inversion).

The PNC interaction in DR mixes the intermediate doubly excited $2 s^{2}$ and $2 s 2 p$ states of the He-like ion, which decay by the emission of a photon. In this respect PNC effects in DR are similar to those in neutron scattering from heavy nuclei. PNC asymmetries of up to $10 \%$ have been observed in nuclei by tuning the neutron energy to the $p$-wave compound nuclear resonances. This enhancement over the typical size of the nuclear weak interaction $\left(10^{-7}\right)$ is caused by the proximity of $s$ - and 
$p$-wave resonances, and by the large ratio of the $s$ - to $p$ wave neutron capture amplitudes (see, e.g., review [12] and references therein).

KLL dielectronic recombination can be observed in experiments with Electron Beam Ion Traps (EBITs) or ion storage rings (see, e.g., [13]). However, these device do not have a polarized ion target or electron beam, as is required. Furthermore, the present generation of devices do not achieve the sensitivity required to observe the PNC effect. Criteria for the sensitivity requirements are outlined in section VI from which the feasibility can be established for any future experimental devices.

The doubly excited $2 l 2 l^{\prime}$ configurations contain a larger number of closely spaced levels than the singly excited $1 s 2 l$ configurations. In Sec. II we calculate the energies of the doubly excited $2 l 2 l^{\prime}$ states for $10 \leq Z \leq 60$ and identify crossings between levels of opposite parity with $\Delta J=0$ and 1 . We then estimate the widths of the close levels of opposite parity and the PNC mixing coefficients $\eta$. In Sec. IV we evaluate the DR cross section and PNC asymmetries. The paper concludes with a short feasibility analysis of PNC measurements in recombination of MCI.

The main aim of our work is to present the first analysis of PNC effects in the DR on H-like ions, to obtain a reliable estimate of the size of the PNC effects and to find the resonances and nuclear charges where these effects are largest. At the next stage it should be possible to improve significantly the accuracy of the calculations by using the well developed theory of the H-like and He-like ions.

\section{ENERGY LEVELS FOR THE $2 l 2 l^{\prime}$ SHELL}

The energies of the $2 l 2 l^{\prime}$ states are determined by diagonalization of the effective Hamiltonian in the $n=2$ subspace. The eigenstates are obtained as $\sum C_{l j, l^{\prime} j^{\prime}}\left|l j, l^{\prime} j^{\prime}\right\rangle$, where $l j$ and $l^{\prime} j^{\prime}$ define the hydrogen-like orbitals with $n=2$. The single-electron part of this Hamiltonian includes hydrogenic Dirac orbital energies and the Lamb shift. The two-electron part of the Hamiltonian matrix for the configurations $2 s^{2}, 2 p^{2}$, and $2 s 2 p$ is taken from Ref. [14. This work presents it as a double expansion in parameters $1 / Z$ and $\alpha Z$ and we use three terms of this expansion of order $Z, Z(\alpha Z)^{2}$, and $Z^{0}$ (atomic units are used throughout the paper and $\alpha \approx 1 / 137$ is the fine structure constant). In particular, the first term accounts for the Coulomb interaction between the electrons. The term $Z(\alpha Z)^{2}$ accounts for the Breit interaction and for the relativistic corrections to the wave functions. The last term $\left(Z^{0}\right)$ corresponds to the second order in the Coulomb interaction.

The Lamb shift appears in the higher order, $Z(\alpha Z)^{3}$, but it is known to be essential for the level crossings within the $1 s 2 l^{\prime}$ manifold [2]. Below we show that this is also true for the $2 l 2 l^{\prime}$ states. By factoring out the main dependence on $Z$ and the principal quantum number $n$,
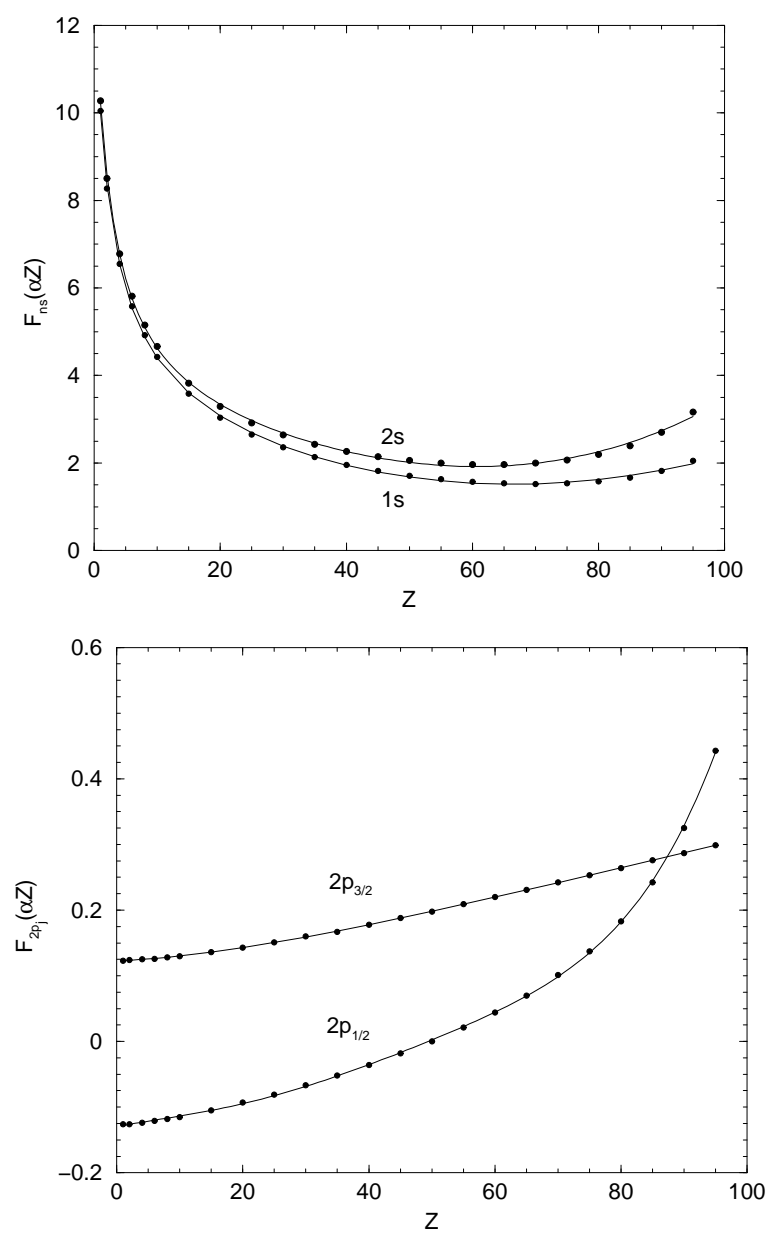

FIG. 1: The Lamb shift for the $1 s, 2 s$, and $2 p_{j}$ orbitals of the H-like ions. Solid circles show the values of $F_{n l j}$ from Eq. (3) calculated by Johnson and Soff [15]. The curves correspond to 5-parameter fits used in our calculations for He-like ions.

the Lamb shift for the hydrogenic orbital $n l j$ is written as

$$
\delta E_{n l j}=\frac{Z(\alpha Z)^{3}}{\pi n^{3}} F_{n l j}(\alpha Z) .
$$

The values of $F_{n l j}$ calculated for $n=1,2$ and $Z$ up to 95 by Johnson and Soff 15 are shown in Fig. 1. They account for the self-energy correction, vacuum polarization, and finite nuclear size effects.

The results of the diagonalization of the Hamiltonian matrix are shown in Fig. 2. The eigenstates are labeled as $\left(2 l 2 l^{\prime}\right)_{J}$ and additional superscripts $a, b$ are added to distinguish levels with identical quantum numbers. One can see two crossings of the levels of opposite parity: a pair of levels with $\Delta J=1$ cross at $Z \approx 17$ and another pair with $\Delta J=0$ cross at $Z \approx 48$. The latter crossing between $\left(2 s^{2}\right)_{0}$ and $(2 s 2 p)_{0}$ levels is entirely due to the Lamb shift. This crossing disappears if the Lamb shift is neglected. Instead, another crossing with $\Delta J=1$ appears near $Z=42$ between the levels $\left(2 p^{2}\right)_{2}^{a}$ and $(2 s 2 p)_{1}^{b}$. As seen in Fig. 2, their energies are very close for $Z>40$. 


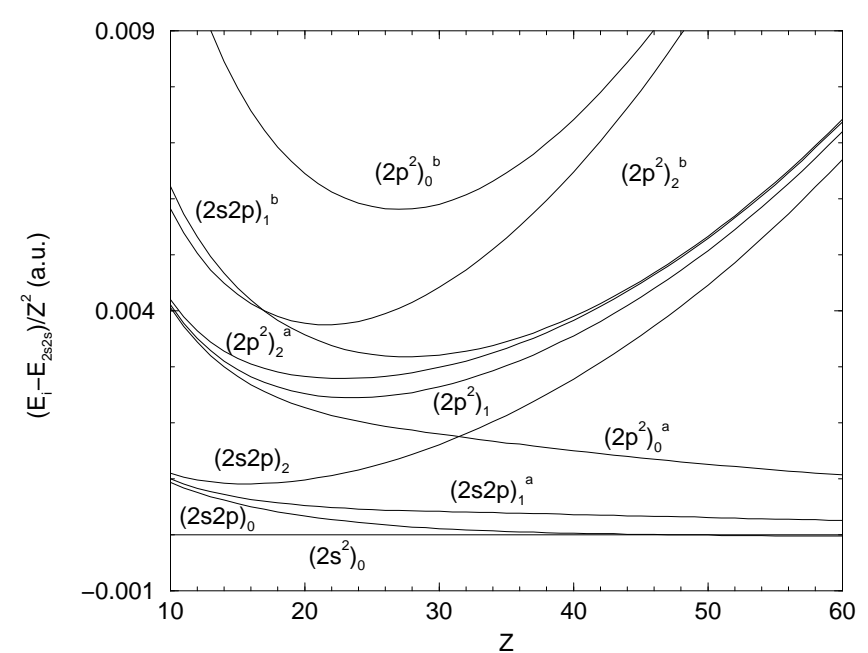

FIG. 2: Energies of the levels of the $2 s 2 p$ and $2 p^{2}$ configurations relative to the $\left(2 s^{2}\right)_{0}$ level for He-like ions. All energies are divided by $Z^{2}$ to account for the general scaling of energies in MCI. The levels are labeled as $\left(2 l 2 l^{\prime}\right)_{J}$ and additional superscripts $a, b$ are added to distinguish levels with identical electron configurations and $J$.

Of course, the exact position of the level crossings may depend on the higher terms of the $\alpha Z$ expansion, which are neglected here. The first crossing at $Z \approx 17$ is rather sharp and takes place at relatively small $Z$, where radiative corrections are still small. Therefore, this crossing is known rather accurately. The crossing between the levels $\left(2 s^{2}\right)_{0}$ and $(2 s 2 p)_{0}$ is more sensitive. However, it should not disappear when higher-order terms are included because of the $Z^{4}$ scaling of the Lamb shift (3). Finally, the relative position of the $\left(2 p^{2}\right)_{2}^{a}$ and $(2 s 2 p)_{1}^{b}$ levels is most sensitive to the higher order terms and they may still cross at high $Z$.

The crossing at $Z \approx 17$ leads to enhanced NSD PNC effects. The crossing at $Z \approx 48$ is favorable for the observation of the NSI part of the PNC interaction. Here much larger PNC effects can be expected due to their scaling with $Z$. In addition, NSI interaction is roughly $Z$ times stronger than NSD interaction, see Sec. IIIA. Because of that we focus on the effects due to PNC mixing of the $\left(2 s^{2}\right)_{0}$ and $(2 s 2 p)_{0}$ levels.

According to Eq. (2) the PNC mixing near the crossing points depends on the line widths. For autoinizing states the total width is the sum of the radiative and autoionizing widths, $\Gamma=\Gamma^{(r)}+\Gamma^{(a)}$. In the non-relativistic hydrogenic approximation the radiative widths of the states $(2 s 2 p)_{0} \equiv(-, 0)$ and $\left(2 s^{2}\right)_{0} \equiv(+, 0)$ are given by:

$$
\begin{aligned}
& \Gamma_{-, 0}^{(r)}=\left(\frac{2}{3}\right)^{8} \alpha^{3} Z^{4}=1.517 \times 10^{-8} Z^{4}, \\
& \Gamma_{+, 0}^{(r)}=2\left(\frac{2}{3}\right)^{8} \alpha^{3} Z^{4}\left(1-C_{s s}^{2}\right),
\end{aligned}
$$

where the coefficient $C_{s s}$ defines contribution of the con- figuration $2 s^{2}$ to the state $|+, 0\rangle$ :

$$
\begin{aligned}
|+, 0\rangle= & C_{s s}|2 s, 2 s\rangle+C_{p p}\left|2 p_{1 / 2}, 2 p_{1 / 2}\right\rangle \\
& +C_{p^{\prime} p^{\prime}}\left|2 p_{3 / 2}, 2 p_{3 / 2}\right\rangle .
\end{aligned}
$$

The autoionizing widths are evaluated in Appendix $\mathrm{A}$. They depend weakly on $Z$ and for $Z>30$ are smaller than the radiative widths. Hence, for $Z>30$ the size of the PNC mixing (2) is limited largely by the radiative width, $\Gamma_{ \pm, 0} \approx \Gamma_{ \pm, 0}^{(r)}$. This means that the exact position of the level crossing is not very important for calculation of the PNC effect.

\section{PNC HAMILTONIAN AND MIXING}

\section{A. PNC Hamiltonian and one-electron PNC matrix element}

The Hamiltonian of the PNC interaction consists of the NSI and NSD parts and in relativistic notation has the form [1]:

$$
\begin{aligned}
H^{\mathrm{PNC}} & =H_{\mathrm{NSI}}^{\mathrm{PNC}}+H_{\mathrm{NSD}}^{\mathrm{PNC}} \\
& =\frac{G_{\mathrm{F}}}{\sqrt{2}}\left(-\frac{Q_{\mathrm{W}}}{2} \gamma_{5}+\frac{\kappa}{I} \gamma_{0} \boldsymbol{\gamma} \cdot \boldsymbol{I}\right) n(\boldsymbol{r}),
\end{aligned}
$$

where $G_{\mathrm{F}}=2.2225 \times 10^{-14}$ a.u. is the Fermi constant of the weak interaction, $\gamma_{i}$ are the Dirac matrices, $\boldsymbol{I}$ is the nuclear spin, and $n(\boldsymbol{r})$ is the nuclear density normalized as $\int n(\boldsymbol{r}) d \boldsymbol{r}=1$. The dimensionless constants $Q_{\mathrm{W}}$ and $\kappa$ characterize the strength of the NSI and NSD parts, respectively. $Q_{\mathrm{W}}$ is known as the weak charge of the nucleus. In the lowest order the standard model yields:

$$
Q_{\mathrm{W}}=-N+Z\left(1-4 \sin ^{2} \theta_{\mathrm{W}}\right) \approx-N,
$$

where $N$ is the number of neutrons and $\theta_{\mathrm{W}}$ is the Weinberg angle, $\sin ^{2} \theta_{\mathrm{W}} \approx 0.23$. Radiative corrections to Eq. (8) change $Q_{\mathrm{W}}$ by few percent [16].

The constant $\kappa$ includes contributions from the anapole moment $\kappa_{\mathrm{a}}$ and from the electron-nucleon neutral currents $\kappa_{e N}\left(\left|\kappa_{e N}\right| \ll 1\right)$. Flambaum and Khriplovich showed that $\kappa_{\mathrm{a}} \sim \alpha A^{2 / 3}$, where $A=Z+N$ is the number of nucleons, and for heavy nuclei dominates over the constant $\kappa_{e N}$ [17. One more contribution to the constant $\kappa$ was calculated by Flambaum and Khriplovich [17] and by Bouchiat and Piketty [18. Except for the very heavy nuclei, this contribution is significantly smaller than that of the anapole moment.

Weak charges of the nuclei ${ }^{203} \mathrm{Tl}$ and ${ }^{133} \mathrm{Cs}$ were measured with high accuracy by Vetter et al. [19] and by Wood et al. 20. These measurements played an important role in low-energy tests of the standard model (see review 21]). Up to now the only measurement of the NSD PNC amplitude was made for ${ }^{133} \mathrm{Cs}$ [20. A detailed discussion of this matter and a complete list of references can be found in the recent review 3 . 
Because of the short-range nature of the interaction in Hamiltonian (7) it effectively mixes only one-electron states with $j=1 / 2$, i.e. $n s_{1 / 2}$ and $\tilde{n} p_{1 / 2}$. For a pointlike nucleus the corresponding matrix element turns to infinity because of the singular behavior of the Dirac orbitals at the origin. For a finite nucleus of the radius $R_{\text {nuc }}$ this matrix element can be approximately given by the following expression [1, 22]:

$$
\begin{aligned}
& \left\langle\tilde{n} p_{1 / 2}\left|H^{\mathrm{PNC}}\right| n s_{1 / 2}\right\rangle= \\
& \quad=-i \frac{\sqrt{2} G_{\mathrm{F}} \alpha Z^{4} R}{8 \pi(\tilde{n} n)^{3 / 2}}\left(Q_{W}+\frac{4 \gamma_{1 / 2}+2}{3} \frac{2}{I}(\boldsymbol{I} \cdot \boldsymbol{j}) \kappa\right)
\end{aligned}
$$

where $R$ is the relativistic enhancement factor:

$$
\begin{aligned}
R & =\frac{4\left(2 Z R_{\mathrm{nuc}} / r_{\mathrm{Bohr}}\right)^{2 \gamma_{1 / 2}-2}}{\Gamma^{2}\left(2 \gamma_{1 / 2}+1\right)}, \\
\gamma_{j} & \equiv\left[(j+1 / 2)^{2}-(\alpha Z)^{2}\right]^{1 / 2},
\end{aligned}
$$

and the following approximation can be used for the nuclear radius:

$$
R_{\text {nuc }}=1.2 A^{1 / 3} \mathrm{Fm}=2.27 \times 10^{-5} A^{1 / 3} .
$$

The accuracy of these expressions is a few percent, at least for the NSI part. A more accurate calculation can easily be done using Dirac orbitals for the finite nucleus. At $1 \%$ level of accuracy the details of the nuclear structure and radiative corrections become important (see [10, 23, 24, 25] and references therein).

\section{B. PNC mixings for He-like ions}

Let us examine the enhancement of the $\mathrm{PNC}$ mixing due to the proximity of the levels of opposite parity in He-like ions. The mixing parameter $\eta$ is estimated with the help of Eqs. (1) and (9). The NSI part of the PNC Hamiltonian mixes only states with $\Delta J=0$. Figure 2 shows that there is only one pair of close levels of opposite parity which meets this requirement, namely $\left(2 s^{2}\right)_{0}$ and $(2 s 2 p)_{0}$. The first of these states has admixtures of the configurations $2 p_{1 / 2}^{2}$ and $2 p_{3 / 2}^{2}$ [see Eq. 60]. For example, for $Z=32$ the weights of these configurations are 0.19 and 0.02 , respectively. Thus, the interaction between configurations $2 s^{2}$ and $2 p^{2}$ should be taken into account. Using Eqs. (6) and (9), we obtain:

$$
\begin{aligned}
& \left\langle-, 0\left|H_{\mathrm{NSI}}^{\mathrm{PNC}}\right|+, 0\right\rangle= \\
& \quad=-i \frac{\sqrt{2} G_{\mathrm{F}}}{64 \pi} \alpha Z^{4} R Q_{W}\left(C_{s s}-C_{p p}\right) \equiv i h^{\mathrm{PNC}} .
\end{aligned}
$$

Results of the calculation of the PNC mixing for different $Z$ are presented in Table I] The resonant enhancement at the level crossing is not very pronounced. Firstly, the level crossing is not sharp. Secondly, for $Z>30$ the radiative width, which grows as $Z^{4}$, becomes greater than the autoionizing width and for $Z>40$ it exceeds

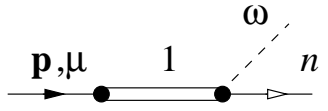

(a)

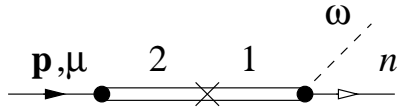

(b)
FIG. 3: Diagrammatic representation of the DR amplitude 15): (a) is the conventional contribution and (b) is the PNC correction. The initial state $\boldsymbol{p}, \mu$ describes the electron with momentum $\boldsymbol{p}$ and helicity $\mu=\boldsymbol{\sigma} \cdot \boldsymbol{p} / 2 p= \pm 1 / 2$ incident on the H-like ion in the $1 s$ ground state. The double lines 1 and 2 correspond to the doubly excited states of the He-like ion which decay by emission of a photon $\omega$ to the final state $n$. The cross denotes PNC mixing of the states 1 and 2 .

the level spacing. As a result, the absolute value of the PNC mixing grows steadily with $Z$. However, the enhancement at the level crossing is clearly seen when we consider the mixing strength divided by the $Z^{3} R$ PNC scaling parameter. The real part of the mixing changes sign at the resonance, where $\eta$ is equal to:

$$
\eta_{Z=48}=(0+6.0 i) \times 10^{-9} \text {. }
$$

\section{PNC EFFECT IN DIELECTRONIC RECOMBINATION}

The formalism we use to calculate the PNC asymmetry in DR is similar to that developed for PNC effects in neutron scattering (see, e.g., review by Flambaum and Gribakin [12]). DR is described by the diagrams in Fig. 3. where we assume that the incident electron $|\boldsymbol{p}, \mu\rangle$ has the energy $\varepsilon$, which is close to the transition energy between the ground state of the H-like ion and the levels $|i=1,2\rangle$ of the configuration $2 l_{i} 2 l_{i}^{\prime}$ of the corresponding He-like ion. Then the contribution of resonance 1 to the DR amplitude $A$ is:

$$
\begin{aligned}
& A \equiv A^{\mathrm{PC}}+A^{\mathrm{PNC}}=\frac{i \sqrt{2 \pi \omega / V}\left\langle n\left|\boldsymbol{e}_{q} \cdot \boldsymbol{r}\right| 1\right\rangle}{E_{1 s}+\varepsilon-E_{1}+\frac{i}{2} \Gamma_{1}} \\
& \left\{\left\langle 1\left|V_{\mathrm{C}}\right| \boldsymbol{p}, \mu ; 1 s_{1 / 2, M}\right\rangle+\frac{\left\langle 1\left|H^{\mathrm{PNC}}\right| 2\right\rangle\left\langle 2\left|V_{\mathrm{C}}\right| \boldsymbol{p}, \mu ; 1 s_{1 / 2, M}\right\rangle}{E_{1 s}+\varepsilon-E_{2}+\frac{i}{2} \Gamma_{2}}\right\},
\end{aligned}
$$

where $\boldsymbol{e}_{q}$ and $\omega$ define the polarization and frequency of the photon, $1 s_{1 / 2, M}$ describes the initial state of the target with spin projection $M$, and $V_{\mathrm{C}}$ is the Coulomb interaction. We use dipole approximation for radiative transition and $V$ is the quantization volume for the electromagnetic field [26]. The total width of the doubly excited states 1 and 2 is given by the sum of the radiative and autoionizing widths, $\Gamma_{i}=\Gamma_{i}^{(r)}+\Gamma_{i}^{(a)}$.

As we have seen in Sec. IIIB, the strongest PNC effect is expected for the crossing of two levels with $J_{1}=J_{2}=$ 0 , which simplifies the derivation. Hence we assume that the states 1 and 2 in Fig. 3 correspond to the levels $| \pm, 0\rangle$ discussed in Sec. IIIB. 
TABLE I: PNC mixing $\eta$ between levels $\left(2 s^{2}\right)_{0}$ and $(2 s 2 p)_{0}$ and comparison of its scaling with typical scaling of the PNC mixing in neutral atoms $\left(Z^{3} R\right)$.

\begin{tabular}{|c|c|c|c|c|c|c|c|c|c|c|c|}
\hline$Z$ & 10 & 15 & 20 & 25 & 30 & 35 & 40 & 45 & 50 & 55 & 60 \\
\hline$\Re \eta \times 10^{11}$ & -0.068 & -0.40 & -1.59 & -6.20 & -19.5 & -58.2 & -159 & -204 & 150 & 383 & 531 \\
\hline$\Im \eta \times 10^{11}$ & 0.004 & 0.018 & 0.076 & 0.41 & 2.2 & 13.0 & 84.3 & 417 & 637 & 620 & 642 \\
\hline$\frac{|\eta|}{Z^{3} R} \times 10^{15}$ & 0.65 & 1.08 & 1.69 & 3.11 & 5.17 & 8.85 & 15.8 & 24.9 & 22.0 & 15.6 & 11.4 \\
\hline
\end{tabular}

The total DR cross section is given by the sum over the final states of the ion and polarization of the photon:

$$
\begin{aligned}
\sigma & \equiv \sigma^{\mathrm{PC}}+\sigma^{\mathrm{PNC}}=\frac{2 \pi}{p} \sum_{q, n} \int\left\{\left|A^{\mathrm{PC}}\right|^{2}+2 \Re\left(A^{\mathrm{PC} *} A^{\mathrm{PNC}}\right)\right\} \\
& \times \delta\left(E_{1 s}+\varepsilon-\omega-E_{n}\right) V d \rho_{\omega},
\end{aligned}
$$

where $d \rho_{\omega}=\omega^{2} d \omega d \Omega /(2 \pi c)^{3}$ and we neglect the square of the small PNC amplitude. When we substitute Eq. 15 in (16), both PC and PNC terms appear to be proportional to the radiative width $\Gamma_{1}^{(r)}$ :

$$
\begin{aligned}
\Gamma_{1}^{(r)} & =2 \pi \sum_{q, n}\left|i \sqrt{2 \pi \omega / V}\left\langle 1\left|\boldsymbol{e}_{q} \cdot \boldsymbol{r}\right| n\right\rangle\right|^{2} \\
& \times \int \delta\left(E_{1 s}+\varepsilon-\omega-E_{n}\right) V d \rho_{\omega} \\
& =\frac{4 \omega^{3} \sum_{n}|\langle n\|r\| 1\rangle|^{2}}{3 c^{3}\left(2 J_{1}+1\right)} .
\end{aligned}
$$

The last expression is standard (see [26]) and was used to calculate $\Gamma_{ \pm, 0}^{(r)}$ in Eqs. (4) and (5). The PC cross section now reads:

$$
\sigma_{1}^{\mathrm{PC}}=\frac{1}{p} \frac{\Gamma_{1}^{(r)}\left|\left\langle\boldsymbol{p}, \mu ; 1 s_{1 / 2, M}\left|V_{\mathrm{C}}\right| 1\right\rangle\right|^{2}}{\left(E_{1 s}+\varepsilon-E_{1}\right)^{2}+\frac{1}{4} \Gamma_{1}^{2}} .
$$

The remaining Coulomb matrix element determines the autoionizing width $\Gamma_{1}^{(a)}$ :

$$
\begin{aligned}
\Gamma_{1}^{(a)} & =\frac{p}{\pi\left(2 J_{1}+1\right)} \sum_{M_{1}, M, \mu}\left|\left\langle\boldsymbol{p}, \mu ; 1 s_{1 / 2, M}\left|V_{\mathrm{C}}\right| 1\right\rangle\right|^{2} \\
& =\frac{2 p}{\pi} \sum_{M}\left|\left\langle\boldsymbol{p}, \mu ; 1 s_{1 / 2, M}\left|V_{\mathrm{C}}\right| 1\right\rangle\right|^{2}
\end{aligned}
$$

where we take into account that $J_{1}=0$. Note that the sum in (19) does not depend on the electron helicity $\mu$, or on the direction of its momentum $\boldsymbol{p}$, while the individual matrix elements do depend on $\mu$ and $M$. Introducing the branching ratio $R_{\mu, M}^{(a)}$ for autoionization into channel $(\mu, M)$, we can rewrite 18 in the final form:

$$
\sigma_{1}^{\mathrm{PC}}=\frac{\pi}{2 p^{2}} \frac{\Gamma_{1}^{(r)} \Gamma_{1}^{(a)} R_{\mu, M}^{(a)}}{\left(E_{1 s}+\varepsilon-E_{1}\right)^{2}+\frac{1}{4} \Gamma_{1}^{2}}
$$

Similarly, the PNC contribution to the cross section becomes:

$$
\sigma_{1}^{\mathrm{PNC}}=\frac{2}{p} \Re\left\{\frac{\Gamma_{1}^{(r)}\left\langle\boldsymbol{p}, \mu ; 1 s_{1 / 2, M}\left|V_{\mathrm{C}}\right| 1\right\rangle\left\langle 1\left|H^{\mathrm{PNC}}\right| 2\right\rangle\left\langle 2\left|V_{\mathrm{C}}\right| \boldsymbol{p}, \mu ; 1 s_{1 / 2, M}\right\rangle}{\left(E_{1 s}+\varepsilon-E_{2}+\frac{i}{2} \Gamma_{2}\right)\left(\left(E_{1 s}+\varepsilon-E_{1}\right)^{2}+\frac{1}{4} \Gamma_{1}^{2}\right)}\right\}=2 \sigma_{1}^{\mathrm{PC}} \Re\left\{\frac{\left\langle 2\left|V_{\mathrm{C}}\right| \boldsymbol{p}, \mu ; 1 s_{1 / 2, M}\right\rangle\left\langle 1\left|H^{\mathrm{PNC}}\right| 2\right\rangle}{\left\langle 1\left|V_{\mathrm{C}}\right| \boldsymbol{p}, \mu ; 1 s_{1 / 2, M}\right\rangle\left(E_{1 s}+\varepsilon-E_{2}+\frac{i}{2} \Gamma_{2}\right)}\right\}
$$

Further simplification of Eq. 21 requires an explicit form of the Coulomb matrix elements. Let us expand the incident electron state in partial waves,

$$
|\boldsymbol{p}, \mu\rangle=\frac{(2 \pi)^{3 / 2}}{\sqrt{p}} \sum_{j, l, m}\left\langle\Omega_{j, l, m}(\hat{\boldsymbol{p}}) \mid \chi_{\mu}(\hat{\boldsymbol{p}})\right\rangle i^{l} e^{i \delta_{j l}}|\varepsilon, j, l, m\rangle
$$

where $\Omega_{j, l, m}$ and $\chi_{\mu}$ are spherical and ordinary spinors and $\delta_{j l}$ is the scattering phase shift. Wave function 22 is normalized so that $\left\langle\boldsymbol{p}^{\prime}, \mu^{\prime} \mid \boldsymbol{p}, \mu\right\rangle=(2 \pi)^{3} \delta\left(\boldsymbol{p}^{\prime}-\boldsymbol{p}\right) \delta_{\mu^{\prime}, \mu}$, and the radial functions are normalized to the delta function of energy, $\left\langle\varepsilon^{\prime}, j^{\prime}, l^{\prime}, m^{\prime} \mid \varepsilon, j, l, m\right\rangle=\delta\left(\varepsilon^{\prime}-\varepsilon\right) \delta_{j^{\prime}, j} \delta_{l^{\prime}, l} \delta_{m^{\prime}, m}$. If we direct the quantization axis along $\hat{\boldsymbol{p}}$, the spinor matrix element in 22 can be written explicitly:

$$
\begin{aligned}
\left\langle\Omega_{j, l, m}(\hat{\boldsymbol{p}}) \mid \chi_{\mu}(\hat{\boldsymbol{p}})\right\rangle & =\sum_{\lambda} C_{l, \lambda, 1 / 2, \mu}^{j, m} Y_{l, \lambda}^{*}(\hat{\boldsymbol{p}}) \\
& =C_{l, 0,1 / 2, \mu}^{j, \mu}\left(\frac{2 l+1}{4 \pi}\right)^{1 / 2} \delta_{m, \mu}
\end{aligned}
$$


where $C_{l, \lambda, s, \mu}^{j, m}$ is the Clebsh-Gordon coefficient and $Y_{l, \lambda}$ is the spherical harmonic.

When we use expansion (22) to calculate the Coulomb matrix elements in Eq. (21), the angular and parity selection rules leave only one term of this expansion with $j=\frac{1}{2}$ and $l=l_{i}=0$, or 1 depending on the parity $P_{i}$ of the intermediate state $i: l_{i}=\left(1-P_{i}\right) / 2$ :

$$
\begin{aligned}
\left\langle i\left|V_{\mathrm{C}}\right| \boldsymbol{p}, \mu ; 1 s_{1 / 2, M}\right\rangle & =\frac{(2 \pi)^{3 / 2}}{\sqrt{p}}\left\langle\Omega_{1 / 2, l_{i},-M}(\hat{\boldsymbol{p}}) \mid \chi_{\mu}(\hat{\boldsymbol{p}})\right\rangle \\
& \times i^{l_{i}} e^{i \delta_{i}}\left\langle P_{i}, 0\left|V_{\mathrm{C}}\right| \varepsilon, \frac{1}{2}, l_{i},-M ; 1 s_{1 / 2, M}\right\rangle,
\end{aligned}
$$

where $\delta_{i} \equiv \delta_{1 / 2, l_{i}}$. Substituting (23) and 24) in (19) we obtain the following expression for the autoionizing width:

$$
\Gamma_{i}^{(a)}=4 \pi\left|\left\langle P_{i}, 0\left|V_{\mathrm{C}}\right| \varepsilon, \frac{1}{2}, l_{i}, \mu ; 1 s_{1 / 2,-\mu}\right\rangle\right|^{2} .
$$

We can also use 23 to find the branching ratio $R_{\mu, M}^{(a)}$ in 20):

$$
R_{\mu, M}^{(a)}=\delta_{\mu,-M} .
$$

This expression is valid only if the quantization axis for the angular momentum of the target ion coincides with the direction of the momentum of the incident electron. Averaging over polarizations of the beam and the target gives $\left\langle R_{\mu, M}^{(a)}\right\rangle=1 / 2$ and Eq. 20 transforms into the standard Breit-Wigner expression [27]:

$$
\sigma_{1}^{\mathrm{PC}}=\frac{\pi}{4 p^{2}} \frac{\Gamma_{1}^{(r)} \Gamma_{1}^{(a)}}{\left(E_{1 s}+\varepsilon-E_{1}\right)^{2}+\frac{1}{4} \Gamma_{1}^{2}} .
$$

Intermediate levels 1 and 2 in Eq. 21) have different parity leading to different partial wave contributing to the matrix element 24): $l_{1}=1-l_{2}$. The corresponding spherical spinors are related by (see, e.g., Ref. [1]):

$$
\Omega_{1 / 2, l_{2}, m}(\hat{\boldsymbol{p}})=-(\boldsymbol{\sigma} \cdot \hat{\boldsymbol{p}}) \Omega_{1 / 2, l_{1}, m}(\hat{\boldsymbol{p}}),
$$

where $(\boldsymbol{\sigma} \cdot \hat{\boldsymbol{p}}) \chi_{\mu}(\hat{\boldsymbol{p}})=2 \mu \chi_{\mu}(\hat{\boldsymbol{p}})$. The partial matrix elements in 24 are real and we can write:

$$
\begin{aligned}
& \frac{\left\langle 2\left|V_{\mathrm{C}}\right| \boldsymbol{p}, \mu ; 1 s_{1 / 2,-\mu}\right\rangle}{\left\langle 1\left|V_{\mathrm{C}}\right| \boldsymbol{p}, \mu ; 1 s_{1 / 2,-\mu}\right\rangle}=-i^{l_{2}-l_{1}} e^{i\left(\delta_{2}-\delta_{1}\right)}(\boldsymbol{\sigma} \cdot \hat{\boldsymbol{p}}) \\
& \quad \times \frac{\left\langle P_{2}, 0\left|V_{\mathrm{C}}\right| \varepsilon, \frac{1}{2}, l_{2}, \mu ; 1 s_{1 / 2,-\mu}\right\rangle}{\left\langle P_{1}, 0\left|V_{\mathrm{C}}\right| \varepsilon, \frac{1}{2}, l_{1}, \mu ; 1 s_{1 / 2,-\mu}\right\rangle} \\
& \quad=-i^{l_{2}-l_{1}} e^{i\left(\delta_{2}-\delta_{1}\right)} \eta_{1,2}(\boldsymbol{\sigma} \cdot \hat{\boldsymbol{p}}) \sqrt{\Gamma_{2}^{(a)} / \Gamma_{1}^{(a)}} .
\end{aligned}
$$

The factor $\eta_{1,2}= \pm 1$ in 30 depends on the signs of the partial matrix elements in 29). Therefore, the PNC cross section (21) takes the form:

$$
\begin{aligned}
\sigma_{1}^{\mathrm{PNC}} & =-2 \eta_{1,2}(\boldsymbol{\sigma} \cdot \hat{\boldsymbol{p}}) \sigma_{1}^{\mathrm{PC}}\left(\frac{\Gamma_{2}^{(a)}}{\Gamma_{1}^{(a)}}\right)^{1 / 2} \\
& \times \Re\left\{e^{i\left(\delta_{2}-\delta_{1}\right)} \frac{i^{l_{2}-l_{1}}\left\langle 1\left|H^{\mathrm{PNC}}\right| 2\right\rangle}{E_{1 s}+\varepsilon-E_{2}+\frac{i}{2} \Gamma_{2}}\right\} .
\end{aligned}
$$

Eq. (31) is valid for the polarized as well as unpolarized target. In the first case one should use Eq. (20), while in the second case Eq. 27) applies. For the unpolarized electron beam $\sigma^{\mathrm{PNC}}$ must be averaged over the helicity and Eq. (31) gives zero for the unpolarized target. However, for the polarized target $\sigma^{\mathrm{PNC}}$ is not zero, because $\sigma^{\mathrm{PC}}$ in Eq. 20 selects the helicity through the branching ratio (26). In fact we can substitute $(\boldsymbol{\sigma} \cdot \hat{\boldsymbol{p}})$ in (31) with $-2(\boldsymbol{S} \cdot \hat{\boldsymbol{p}})$, where $\boldsymbol{S}$ is the spin of the ion.

\section{RESULTS}

Now we apply the formalism developed in the previous sections to calculate the PNC effect in the DR cross section at the energies near the $( \pm, 0)$ resonances in the He-like ions. In the diagrams in Fig. 3 and in corresponding equations (27) and (31) the states 1 and 2 can be either $\left(2 s^{2}\right)_{0}$ and $(2 s 2 p)_{0}$, or vice versa. These two contributions lead to the final states $n$ with different parities and we sum the corresponding cross sections, $\sigma^{\mathrm{PC}}=\sigma_{1}^{\mathrm{PC}}+\sigma_{2}^{\mathrm{PC}}$.

Equations (A3), (A5), and A6 show that the phase factor $\eta_{1,2}$ in (31) is equal to 1 . The incident electron energy scales as $Z^{2}$ and the Coulomb phase shifts in the non-relativistic approximation are independent of $Z$, $\delta_{s p} \equiv \delta_{s}-\delta_{p} \approx 0.953$. Taking this into account and using (27) and (31), we obtain the following total PNC cross section:

$$
\begin{aligned}
\sigma^{\mathrm{PNC}}= & -\frac{\pi(\boldsymbol{\sigma} \cdot \hat{\boldsymbol{p}}) \sqrt{\Gamma_{+}^{(a)} \Gamma_{-}^{(a)}} h^{\mathrm{PNC}}}{2 p^{2}\left(\Delta_{+}^{2}+\frac{1}{4} \Gamma_{+}^{2}\right)\left(\Delta_{-}^{2}+\frac{1}{4} \Gamma_{-}^{2}\right)} \\
& \times\left[\left(\Gamma_{+}^{(r)} \Delta_{-}+\Gamma_{-}^{(r)} \Delta_{+}\right) \cos \delta_{s p}\right. \\
& \left.-\frac{1}{2}\left(\Gamma_{+}^{(r)} \Gamma_{-}-\Gamma_{-}^{(r)} \Gamma_{+}\right) \sin \delta_{s p}\right],
\end{aligned}
$$

where $\Delta_{ \pm} \equiv E_{1 s}+\varepsilon-E_{ \pm}, h^{\mathrm{PNC}}$ is given by $(13)$, and again we can substitute $(\boldsymbol{\sigma} \cdot \hat{\boldsymbol{p}})$ with $-2(\boldsymbol{S} \cdot \hat{\boldsymbol{p}})$ for a polarized target rather than a polarized electron beam.

Figure 4 presents the plots of $\sigma^{\mathrm{PC}}, \sigma^{\mathrm{PNC}}$ and the PNC asymmetry,

$$
\mathcal{A}=\left.\frac{\sigma^{+}-\sigma^{-}}{\sigma^{+}+\sigma^{-}} \simeq \frac{\sigma^{\mathrm{PNC}}}{\sigma^{\mathrm{PC}}}\right|_{\boldsymbol{\sigma} \cdot \hat{p}=1},
$$

where $\sigma^{ \pm}$are the cross sections for positive and negative helicity. The peak magnitude of the asymmetry increases from $3 \times 10^{-10}$ for $Z=30$ to $5 \times 10^{-9}$ for $Z=48$, i.e. at the crossing point. It practically does not grow at larger $Z$. Figure 4 also shows that for $Z \geq 40$ the two resonances overlap.

For $Z>30$ the radiative width dominates over the autoionizing width and second term in square brackets in 32 is suppressed. The first term changes sign between the resonances. Therefore, the PNC cross section and asymmetry also change sign. Consequently, the net asymmetry integrated over energy is suppressed. Growth 

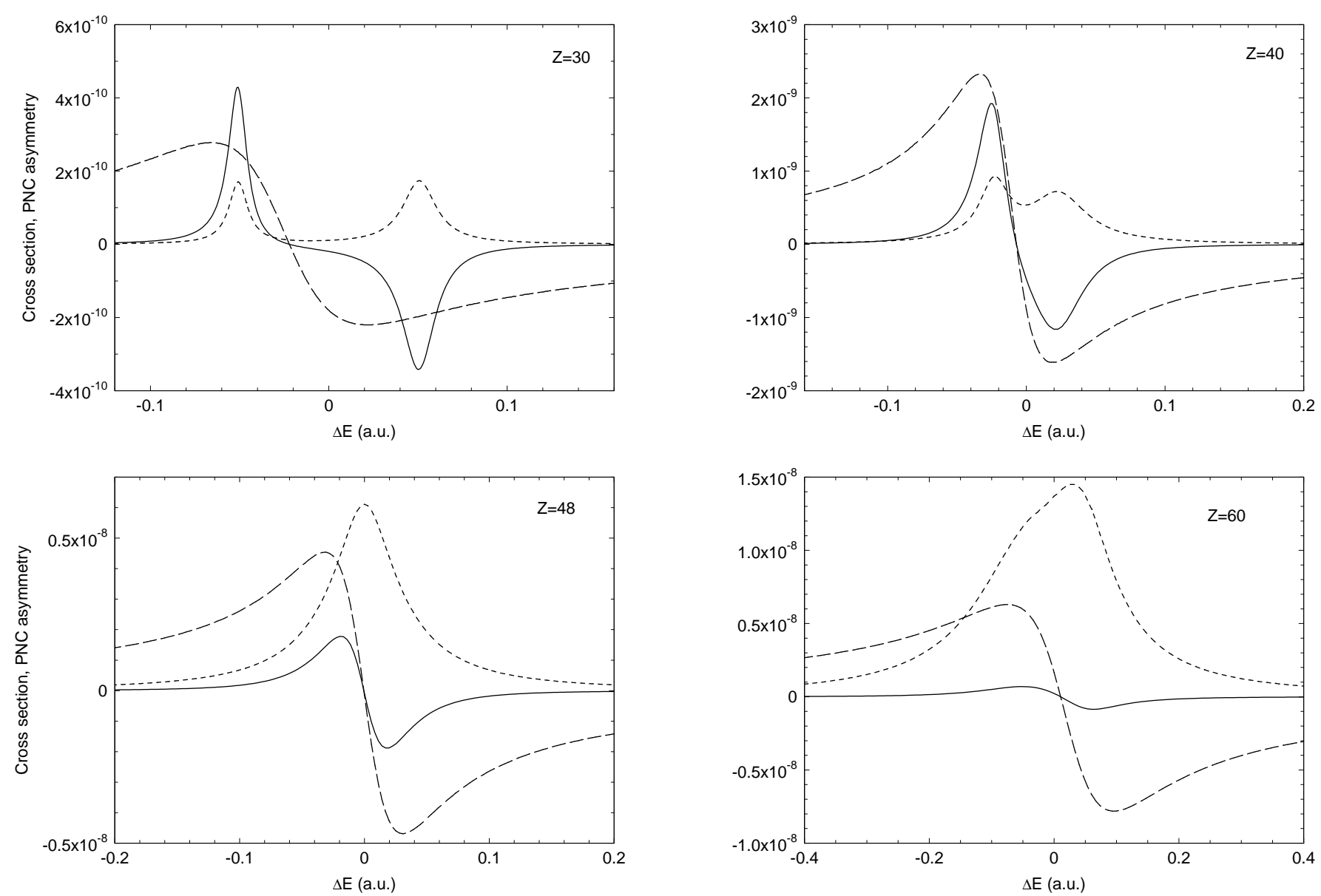

FIG. 4: PC and PNC DR cross sections (in a.u.) and PNC asymmetry for $\left(2 s^{2}\right)_{0}$ and $(2 s 2 p)_{0}$ resonances in H-like ions with $Z=30,40,48$, and 60 . The energy $\Delta E=\varepsilon-E_{\mathrm{av}}+Z^{2} / 2$, where $\varepsilon$ and $-Z^{2} / 2$ are the energies of the incident electron and H-like target and $E_{\mathrm{av}}=\left(E_{\left(2 s^{2}\right)_{0}}+E_{(2 s 2 p)_{0}}\right) / 2$. Solid lines correspond to $10^{3} \times\left.\sigma^{\mathrm{PNC}}\right|_{\boldsymbol{\sigma} \cdot \hat{\boldsymbol{p}}=1}$, long-dashed lines are the PNC asymmetry $\mathcal{A}$, and short-dashed lines correspond to $10^{-n} \times \sigma^{\mathrm{PC}}$, where $n=7,6,5,4$ for $Z=30,40,48,60$.

of the PNC matrix element for higher $Z$ is compensated by the increase in the width and PNC asymmetry stops growing.

\section{DISCUSSION AND CONCLUSIONS}

It is useful to estimate the feasibility of measuring the PNC asymmetry in KLL recombination calculated above and from this estimate derive the sensitivity requirements for an experimental apparatus capable of observing the PNC using the scheme proposed. The number of counts in an experiment with a fully polarized electron beam with positive helicity is given by:

$$
N_{+}=j_{e} N_{i} t \epsilon \sigma^{+} \equiv I \sigma^{+},
$$

where $j_{e}$ is the electron flux, $N_{i}$ is the number of target ions, $t$ is the acquisition time, and $\epsilon$ is the detection efficiency. The number of counts for negative helicity is $N_{-}=I \sigma^{-}$.
For a beam or target with polarization $P$, to detect the PNC asymmetry, the difference between the counts, $P\left|N_{+}-N_{-}\right|$should be greater than statistical error, $\sqrt{N_{+}+N_{-}}$, which gives:

$$
I>\frac{\sigma^{+}+\sigma^{-}+2 \sigma_{b}}{P^{2}\left(\sigma^{+}-\sigma^{-}\right)^{2}},
$$

where $\sigma_{b}$ is the magnitude of any background occurring through direct radiative recombination or as an experimental artifact (e.g. detector dark counts). We express this as a cross section for convenience although for experimental artifact background signal, this is the effective cross section to which the apparatus background would correspond. For the rest of this analysis we consider the ideal limit, $P=1, \sigma_{b}=0$.

Equation (35) is valid for a mono-energetic electron beam. If the electron energy spread in the beam is greater than the resonance spacing and widths, then the flux $j_{e}$ in (34) should be replaced by the flux density $d j_{e} / d \varepsilon$. The counts $N_{ \pm}$are obtained by integrating over the electron 
energy and the effect can be detected if

$$
I_{\mathrm{av}}>\int\left(\sigma^{+}+\sigma^{-}\right) d \varepsilon\left(\int\left(\sigma^{+}-\sigma^{-}\right) d \varepsilon\right)^{-2} .
$$

The first integral above is equal to $2\left(S_{1}+S_{2}\right)$, where

$$
S_{i}=\frac{\pi^{2}}{2 p^{2}} \frac{\Gamma_{i}^{(r)} \Gamma_{i}^{(a)}}{\Gamma_{i}}
$$

is the strength of resonance $i$. The integral $\int\left(\sigma^{+}-\sigma^{-}\right) d \varepsilon$ in Eq. (36) can be written as $2 S_{1,2}^{\mathrm{PNC}}$, where

$$
\begin{aligned}
S_{1,2}^{\mathrm{PNC}} \equiv & \left.\int \sigma^{\mathrm{PNC}}\right|_{\boldsymbol{\sigma} \cdot \hat{\boldsymbol{p}}=1} d \varepsilon \\
= & -\frac{\pi^{2}}{p^{2}} \frac{\sqrt{\Gamma_{+}^{(a)} \Gamma_{-}^{(a)}} h^{\mathrm{PNC}}\left(\frac{\Gamma_{+}^{(r)}}{\Gamma_{+}}-\frac{\Gamma_{-}^{(r)}}{\Gamma_{-}}\right)}{\left[\left(E_{+}-E_{-}\right)^{2}+\frac{1}{4}\left(\Gamma_{+}+\Gamma_{-}\right)^{2}\right]} \\
& \times\left[\left(E_{+}-E_{-}\right) \cos \delta_{s p}-\frac{1}{2}\left(\Gamma_{+}+\Gamma_{-}\right) \sin \delta_{s p}\right],
\end{aligned}
$$

is the PNC strength of the two resonances. Thus, Eq. (36) reads:

$$
I_{\text {av }}>\frac{1}{2}\left(S_{1}+S_{2}\right) /\left(S_{1,2}^{\mathrm{PNC}}\right)^{2} .
$$

Equations (35) and (39) show that for the two limiting cases of narrow and wide energy distribution in the beam the feasibility of the experiment on ions with nuclear charge $Z$ depends on the functions:

$$
\begin{aligned}
F & =\min \left[\left(\sigma^{+}+\sigma^{-}\right) /\left(\sigma^{+}-\sigma^{-}\right)^{2}\right], \\
F_{\mathrm{av}} & =\int\left(\sigma^{+}+\sigma^{-}\right) d \varepsilon\left[\int\left(\sigma^{+}-\sigma^{-}\right) d \varepsilon\right]^{-2},
\end{aligned}
$$

where minimum is taken with respect to the energy of the beam. These functions are shown in Fig. 5, where cross sections are in barns $\left(10^{-24} \mathrm{~cm}^{2}\right)$ and energy in $\mathrm{eV}$.

For mono-energetic beam the measurements become more feasible at large $Z$, where the PNC asymmetry is greater. The strongest effect can be seen for $Z \approx 45$, i.e. near the level crossing. The averaged PNC effect is strongly suppressed by the factor $\left(\Gamma_{+}^{(r)} / \Gamma_{+}-\Gamma_{-}^{(r)} / \Gamma_{-}\right)$ in Eq. (38), which monotonically decreases with $Z$, as $\Gamma_{ \pm}^{(r)} / \Gamma_{ \pm} \rightarrow 1$. This suppression is caused by almost antisymmetric shape of the PNC signal along the resonances. We conclude that observation of the PNC effects in DR is much easier with mono-energetic beam.

It is worth noting that $F^{-1}$ and $F_{\text {av }}^{-1}$ have the same dimensions as a cross section and a resonance strength respectively. Indeed, these quantities are useful for estimating the feasibility of any future experiment designed to observe the PNC effect in KLL dielectronic recombination resonances. For an experiment to be able to observe the effect predicted, it would have to be able to detect a cross section as small as $F^{-1}$ or a resonance strength as small as $F_{\mathrm{av}}^{-1}$, i.e. about $10^{-12} \mathrm{~b}$ or $10^{-15} \mathrm{~b} \mathrm{eV}$, in the absence of background. Of course, this is extremely demanding, but it is worth remarking that the level crossing

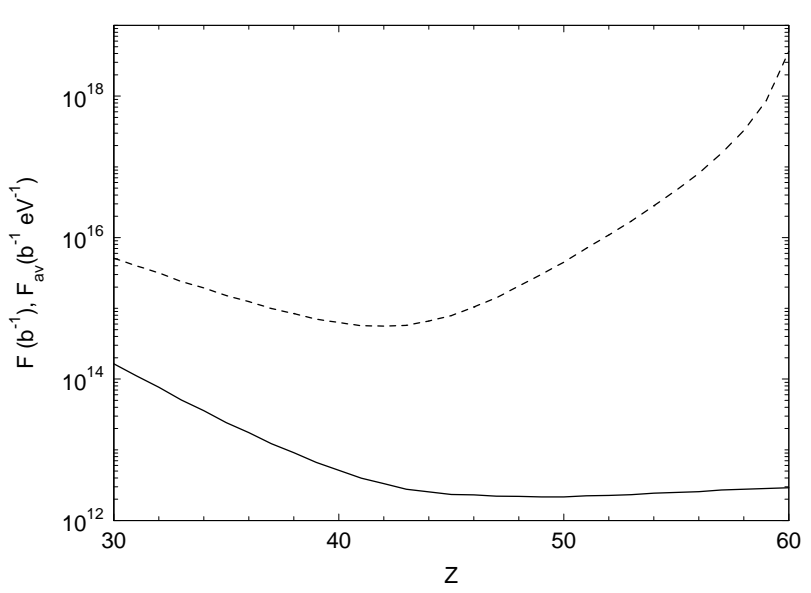

FIG. 5: PNC measurement feasibility function $F$ in $\mathrm{b}^{-1}$ (solid line) and $F_{\text {av }}$ in $\mathrm{b}^{-1} \mathrm{eV}^{-1}$ (dashed line). Shallow minimum of $F$ at $Z \approx 45$ approximately corresponds to the level crossing.

considered here, gives rise to an enhancement of eight orders of magnitude compared to the basic strength of the weak interaction in atoms.

Let us compare the present scheme with other proposals for measuring PNC in ions. Pindzola [6] suggested to observe PNC effect in the Auger emission from the He-like uranium. He considered the mixing of the same states, $2 s^{2}$ and $2 s 2 p$ with $J=0$, and obtained asymmetries of about $10^{-7}$, which is comparable to our results. However, that estimate neglected the radiative widths of the levels, which for $Z \gtrsim 50$ exceed the level spacing.

Other proposals were based on the observations of $\mathrm{PNC}$ asymmetries in radiative transitions in He-like ions. Schafer et al. 44 focused on the two-photon E1-M1 transition between two metastable levels, $2{ }^{3} P_{0} \rightarrow 2{ }^{1} S_{0}$, separated by $1 \mathrm{eV}$ in $\mathrm{U}^{90+}$. They showed that the PNC mixing is $|\eta| \sim 5 \times 10^{-6}$, and concluded that lasers with intensities above $10^{21} \mathrm{~W} / \mathrm{cm}^{2}$ would be required to observe it. In Refs. [5, 7, 8, two-photon and hyperfinequenched transitions $2{ }^{1} S_{0} \rightarrow 1{ }^{1} S_{0}$ were examined. Here the mixing between $2{ }^{1} S_{0}$ and $2{ }^{3} P_{0}$ levels leads to circular polarization of the photons (up to $10^{-4}$ ) or to an asymmetry in the photon angular distribution $\left(4 \times 10^{-4}\right.$ for $\mathrm{Gd}^{62+}$ for a fully polarized ion beam). Although these values seem large, there is a number of associated problems: low counting rates for the highly forbidden transitions involved, photon background, detection of the circular polarization of gamma quanta, and creation of the polarized ion beam. As a result, the number of events necessary to measure the effect is large, e.g. $\sim 10^{18}[9$.

\section{Acknowledgments}

Authors are thankful to V. Shabaev, who pointed at the sign error in the numerator of Eq. $\sqrt{38}$ in the published version of this paper 29]. In this corrected version of the e-print we traced the error back to Eq. (30) and 
made respective changes to the text and figures.

Authors are grateful to A. Korol, L. Labzowsky, and A. Nefiodov for valuable discussions and to D. Budker for reading the manuscript. This work is supported in part by Russian Foundation for Basic Research, grant No. 0502-16914. One of us (MK) thanks International Research Centre for Experimental Physics in Belfast for the DVF fellowship.

\section{APPENDIX A: CALCULATION OF AUTOIONIZING WIDTHS $\Gamma_{ \pm, 0}^{(a)}$}

The autoionizing widths of the doubly excited states with $J=0$ are given by Eq. (25). Their wave functions are linear combinations of the two-electron states of the form:

$$
\left|P, 0\left(l_{b}, l_{c}\right)\right\rangle=\sum_{m} \frac{(-1)^{j-m}}{\sqrt{2 j+1}}\left|2, l_{b}, j, m\right\rangle\left|2, l_{c}, j,-m\right\rangle,
$$

where parity $P=(-1)^{l_{b}+l_{c}}$. In the initial state the incident electron is described by the wave function 22 . and the H-like ion is in the ground state $\left|1 s_{1 / 2, M}\right\rangle$. The Coulomb matrix elements on the right-hand-side of (24), after substituting (A1), are reduced to the two-electron matrix elements:

$$
\begin{aligned}
& \left\langle 2 l_{b}, j_{b}, m_{b} ; 2 l_{c}, j_{c}, m_{c}\left|V_{\mathrm{C}}\right| \varepsilon, l_{i}, j_{i}, m_{i} ; 1 s, \frac{1}{2}, m\right\rangle \\
& =(-1)^{m_{c}+m_{i}+1}\left[j_{b}, j_{c}, j_{i}, \frac{1}{2}\right] \sum_{K}\left(\begin{array}{ccc}
j_{i} & j_{b} & K \\
-m_{i} & m_{b} & Q
\end{array}\right) \quad(\mathrm{A} 2) \\
& \times\left(\begin{array}{ccc}
j_{c} & \frac{1}{2} & K \\
-m_{c} & m & Q
\end{array}\right)\left(\begin{array}{ccc}
j_{i} & j_{b} & K \\
\frac{1}{2} & -\frac{1}{2} & 0
\end{array}\right)\left(\begin{array}{ccc}
j_{c} & \frac{1}{2} & K \\
\frac{1}{2} & -\frac{1}{2} & 0
\end{array}\right) R_{b, c, i, 1 s}^{K},
\end{aligned}
$$

where $\left[j_{a} \ldots\right] \equiv\left[\left(2 j_{a}+1\right) \ldots\right]^{1 / 2}$ and $R_{a, b, c, d}^{K}$ is the Coulomb radial integral. It is nonzero for even $K+l_{a}+l_{c}$ and $K+l_{b}+l_{d}$. Equations (A1) and (A2) allow one to calculate the matrix elements in 24). Neglecting the dependence of the radial integrals on $j$ we have for the odd state,

$$
\begin{aligned}
& \left\langle-1,0\left|V_{\mathrm{C}}\right| \varepsilon, \frac{1}{2}, l_{i},-m ; 1 s_{1 / 2, M}\right\rangle \\
& \quad=\delta_{l_{i}, 1} \frac{\tau_{m}}{\sqrt{2}}\left(R_{2 p, 2 s, \varepsilon p, 1 s}^{0}-\frac{1}{3} R_{2 s, 2 p, \varepsilon p, 1 s}^{1}\right),
\end{aligned}
$$

where $\tau_{m} \equiv(-1)^{m+1 / 2}$. For the even state A1 we obtain

$$
\begin{aligned}
& \left\langle+1,0\left(2 l^{2}\right)\left|V_{\mathrm{C}}\right| \varepsilon, \frac{1}{2}, l_{i},-m ; 1 s_{1 / 2, M}\right\rangle \\
& =\delta_{l_{i}, 0}(-1)^{l} \tau_{m} \frac{(j+1 / 2)^{1 / 2}}{2 l+1} R_{2 l, 2 l, \varepsilon s, 1 s}^{l},
\end{aligned}
$$

and for the eigenstate $(6)$ we arrive at

$$
\begin{aligned}
& \left\langle+1,0\left|V_{\mathrm{C}}\right| \varepsilon, \frac{1}{2}, 0,-m ; 1 s_{1 / 2, M}\right\rangle \\
& =\tau_{m}\left(C_{s s} R_{2 s, 2 s, \varepsilon s, 1 s}^{0}-\frac{C_{p p}+\sqrt{2} C_{p^{\prime} p^{\prime}}}{3} R_{2 p, 2 p, \varepsilon s, 1 s}^{1}\right) .
\end{aligned}
$$

To estimate the widths $\Gamma_{i}^{(a)}$ we use non-relativistic hydrogenic radial Coulomb integrals, which do not depend on $Z$ :

$$
\begin{array}{cccc}
R_{2 s, 2 s, \varepsilon s, 1 s}^{0} & R_{2 p, 2 s, \varepsilon p, 1 s}^{0} & R_{2 s, 2 p, \varepsilon p, 1 s}^{1} & R_{2 p, 2 p, \varepsilon s, 1 s}^{1} \\
0.0200 & -0.0304 & 0.0310 & -0.0300
\end{array}
$$

Equations (25), (A3), and (A6) give

$$
\Gamma_{-, 0}^{(a)}=0.0104
$$

for all $Z . \Gamma_{+, 0}^{(a)}$ is somewhat smaller than $\Gamma_{-, 0}^{(a)}$ and weakly depends on $Z$ via the coefficients $C_{a a}$ in Eq. A5. For pure $\left(2 s^{2}\right)_{0,0}$ state Eqs. 25) and (A4) give $\Gamma_{2 s^{2}}^{(a)}=$ 0.00496. This value is in agreement with Ref. [28].

The same nonrelativistic hydrogenic approximation for the radiative transitions was used in Eqs. (4) and (5) for the radiative widths $\Gamma_{ \pm, 0}^{(r)}$. Again the negative parity state has larger width. Comparison of Eqs. A7) and (4) shows that the radiative width becomes equal to the autoionizing width for $Z \approx 29$ and dominates near the level crossing at $Z \approx 48$.
[1] I. B. Khriplovich, Parity non-conservation in atomic phenomena (Gordon and Breach, New York, 1991).

[2] V. G. Gorshkov and L. N. Labzovskii, Pis'ma v ZhETF 19, 768 (1974).

[3] J. S. M. Ginges and V. V. Flambaum, Phys. Rep. 397, 63 (2004).

[4] A. Schafer, G. Soff, P. Indelicato, B. Müller, and
W. Greiner, Phys. Rev. A 40, 7362 (1989).

[5] V. V. Karasiov, L. N. Labzowsky, and A. V. Nefiodov, Phys. Lett. A 172, 62 (1992).

[6] M. S. Pindzola, Phys. Rev. A 47, 4856 (1993).

[7] R. W. Dunford, Phys. Rev. A 54, 3820 (1996).

[8] L. N. Labzowsky et al., Phys. Rev. A 63, 054105 (2001).

[9] A. V. Nefiodov, L. N. Labzowsky, D. Liesend, G. Plunien, 
and G. Soff, Phys. Lett. B 534, 52 (2002).

[10] A. I. Milstein and O. P. Sushkov (2004), E-print:hep$\mathrm{ph} / 0409149$.

[11] O. Y. Andreev, L. N. Labzowsky, G. Plunien, and G. Soff, Nucl. Instrum. and Methods B 205, 25 (2003).

[12] V. V. Flambaum and G. F. Gribakin, Prog. Part. Nucl. Phys. 35, 423 (1995).

[13] E. F. Currell, ed., The Physics of Multiply and Highly Charged Ions: Vol.1 Sources, Applications and Fundamental Processes (Kluwer Academic Publishers, Dordrecht, The Netherlands, 2003), ISBN 1-4020-1565-8.

[14] M. A. Braun, A. D. Gurchumelia, and U. I. Safronova, Relativistic Theory of Atoms (Nauka, Moscow, 1984), (in Russian).

[15] W. R. Johnson and G. Soff, Atomic Data and Nuclear Data Tables 33, 405 (1985).

[16] K. Hagiwara et al., Phys. Rev. D 66, 010001 (2002).

[17] V. V. Flambaum and I. B. Khriplovich, Sov. Phys.-JETP 52, 835 (1980)

[18] C. Bouchiat and C. A. Piketty, Phys. Lett. B 269, 195 (1991), Erratum: 274, 526 (1992).

[19] P. A. Vetter, D. M. Meekhof, P. K. Majumder, S. K. Lamoreaux, and E. N. Fortson, Phys. Rev. Lett. 74, 2658
(1995).

[20] C. S. Wood, S. C. Bennett, D. Cho, B. P. Masterson, J. L. Roberts, C. E. Tanner, and C. E. Wieman, Science 275, 1759 (1997).

[21] J. Erler and M. J. Ramsey-Musolf (2004), E-print:hep$\mathrm{ph} / 0404291$.

[22] M. A. Bouchiat and C. Bouchiat, Phys. Lett. B 48, 111 (1974).

[23] M. Y. Kuchiev and V. V. Flambaum, J. Phys. B 36, R191 (2003).

[24] A. I. Milstein and O. P. Sushkov, Phys. Rev. A 66 , 022108 (2002).

[25] A. Derevianko and S. G. Porsev, Phys. Rev. A 65, 052115 (2002).

[26] I. I. Sobelman, Atomic spectra and radiative transitions (Springer-Verlag, Berlin, 1979).

[27] L. D. Landau and E. M. Lifshitz, Quantum mechanics (Pergamon, Oxford, 1977), 3rd ed.

[28] M. Y. Amusia, A. I. Mikhailov, and I. A. Mikhailov, Sov. Phys.-JETP 86, 299 (1998).

[29] G. F. Gribakin, F. J. Currell, M. G. Kozlov, and A. I. Mikhailov, Phys. Rev. A 72, 032109 (2005). 